\title{
Atomic force microscopy of polymer crystals: 7. Chain packing, disorder and imaging of methyl groups in oriented isotactic polypropylene
}

\author{
Daniel Snétivy and G. Julius Vancso* \\ University of Toronto, Department of Chemistry, 80 St George Street, Toronto, Ontario \\ M5S 1A1, Canada \\ (Received 3 February 1993; revised 24 April 1993)
}

\begin{abstract}
Atomic force microscopy (AFM) was used to study the morphology and nanostructure of mechanically oriented, isotactic polypropylene (i-PP). AFM images of cleaved samples on the micrometre scale unveiled microfibrils with a diameter of $100-150 \mathrm{~nm}$. Chain orientation and imperfections of chain packing were visualized in the $(110)$ crystal facet at the surface of the microfibrils. Paracrystalline disorder and twisting of macromolecules were observed. Resolution of individual methyl groups with unprecedented quality allowed us to identify the handedness of the helices in the crystalline i-PP matrix. The packing of the methyl groups, visualized by AFM, was compared with the computer-visualized structure. The agreement between the expected and the experimentally determined parameters of the crystal structure was perfect within experimental accuracy.
\end{abstract}

(Keywords: atomic force microscopy; molecular orientation; isotactic polypropylene)

\section{INTRODUCTION}

Atomic force microscopy $(\mathrm{AFM})^{1-4}$ has been used with great success to image the macromolecular architecture of polymeric materials on the angstrom scale ${ }^{4-12}$, as well as to visualize morphological details in the micrometre domain $^{14,15}$. Thus, the technique is capable of covering a magnification range of $\approx 10^{8}-10^{3}$. When used to study the atomic and molecular structures of surfaces, AFM is complementary to X-ray diffraction since it yields local information on the structure in the direct space. Data obtained by diffraction techniques represent averages of the structural parameters over $\approx 10^{20}$ atoms. AFM, on the other hand, can be used to visualize as few as several dozen atoms or groups of atoms at preselected locations on the sample surface. This unique feature of AFM allows local information to be obtained about the molecular organization of the bulk polymer since molecular relaxation of polymers with small substituents is likely to be negligibly small ${ }^{9}$ on the cleaved surfaces of crystals. In addition, conformation of individual chains can also be studied in the solid state ${ }^{6,12}$.

Owing to the novelty of the technique and the lack of a comprehensive understanding of the imaging process, the majority of the papers on the AFM of polymers published so far include observational work and confirm known results. In a few cases, however, problems that it was not possible to tackle prior to the advent of AFM have been successfully addressed. For example, the parameters of a novel crystal structure of a new

\footnotetext{
* To whom correspondence should be addressed
}

polymorphic form in poly ( $p$-phenylene terephthalamide) (PPTA, Kevlar ${ }^{(\mathbb{R}}$ ) fibres have been determined ${ }^{11}$. This new structure of PPTA had been suggested by computer simulations ${ }^{16}$, but because of the simultaneous coexistence of different polymorphic forms in non-annealed fibres, it was not possible to confirm its existence by X-ray diffraction.

Our earlier work demonstrated that AFM is very successful at imaging macromolecules in ordered (oriented) systems. The technique has, therefore, the potential to address questions related to the orientation mechanisms and the short-range versus long-range order and disorder in anisotropic polymers. In this paper we present AFM results of a mechanically oriented, commercial, isotactic polypropylene (i-PP) sample with unprecedented image quality. This has been achieved by a careful control of the humidity in the compartment of the scan head. Individual methyl groups are resolved in raw-data images which allow one to obtain an insight into the alignment, handedness, packing and twisting of macromolecular chains within the microfibrillar crystals formed during the mechanical orientation process. Thus, we obtained information about the structural order and disorder in the crystalline phase.

Isotactic PP was the subject of early AFM studies? Oligomers of i-PP were epitaxially crystallized on benzoic acid crystals. AFM was used to study the surface of polymer crystals grown in contact with the substrate after the dissolution of benzoic acid in hot methanol. It was concluded that the crystal facet of polypropylene, grown in contact with the substrate, was the $\left(\begin{array}{lll}0 & 1 & 0\end{array}\right)$ plane, and it consisted of left-handed helices. Fourier inversion and 
filtering were performed in this work to improve the AFM image quality. The raw-data AFM nanographs were not included in the original publication.

We have already reported on our preliminary AFM results ${ }^{11}$ including molecular resolution obtained on mechanically oriented, commercial i-PP. The polymer used in this study was a commercial sample, and thus information obtained on the chain orientation and molecular structure can also be of practical interest. AFM results showed microfibrils with a typical average diameter of $150 \mathrm{~nm}$. High magnification scans unveiled individual macromolecules with a $1.17 \mathrm{~nm}$ chain-chain packing distance, which was consistent with the imaging of the $\left(\begin{array}{ll}1 & 10)\end{array}\right)$ crystal facet. On the nanographs either ridges corresponding to the macromolecular chains, or rowlike features at angles of $40^{\circ}$ with respect to the chain direction, were observed. These different features were observed depending on the orientation of the scan direction of the pyramidal AFM tip with respect to the polymeric fibre (and thus also with the expected chain direction). We concluded that the rowlike features at angles of $40^{\circ}$ with respect to the chain direction, which sometimes completely masked the image of the chains, correspond to rows formed of neighbouring methyl groups of the main chain. In this early work no resolution of the individual methyl groups was observed on the raw-data images. In the present work, detailed experimental data are presented which confirm the aforementioned interpretation of our AFM images of i-PP.

\section{EXPERIMENTAL}

Specimens were prepared from commercially available Propathene ${ }^{\circledR}$ GYM 45 isotactic PP polymer (ICI, batch no. 93996). Orientation was carried out by using standard tensile test samples which were obtained by injection moulding. Specimens were uniaxially stretched in a tensile test instrument (Schenk-Trebel, model RM-100) at a temperature of $120^{\circ} \mathrm{C}$, and at a strain rate of $10 \mathrm{~mm} \mathrm{~min}^{-1}$ until an elongation ratio of $l / l_{0}=6$ was reached. The samples were then quenched under constant stress to prevent relaxation.

Samples for AFM studies were prepared from oriented i-PP at ambient temperatures by using a Sorvall MT6000 ultramicrotome with glass knives. Specimens were cut in planes containing the orientation direction. Precise microtome settings and careful operation ensured that the microfibrils were cleaved, and thus damage to the structure of the material was avoided. AFM images were taken in air using a NanoScopeII (Digital Instruments, Santa Barbara, CA, USA) instrument. Molecular resolution was obtained by an 'A-type' scan head utilizing NanoProbe 100 micrometre triangular $\mathrm{Si}_{3} \mathrm{~N}_{4}$ cantilevers (Digital Instruments) with narrow legs. The effective spring constant of the cantilever, as specified by the supplier, was $0.38 \mathrm{~N} \mathrm{~m}^{-1}$. Images of the microfibrils were obtained by a 'D-head' using cantilevers with long and narrow legs (spring constant: $0.12 \mathrm{~N} \mathrm{~m}^{-1}$ ). Images obtained on the surface of muscovite mica (J.B. EM Services Inc., Pointe-Claire, Québec, Canada) with 'molecular' resolution were used for calibration of the piezo controller in the $x-y$ plane of the scans.

It is known that humidity has a great influence on AFM image quality. This is probably due to many factors such as the influence of the adsorbed water layer at the sample surface on the imaging mechanism, elimination of static charges for non-conducting surfaces, etc. We have not found a relationship between the image quality obtained on various surfaces as a function of humidity. It seems that mica and graphite yield good quality AFM images on the molecular level in relative air humidities between 40 and $70 \%$. On the other hand, the best image quality for polypropylene with molecular resolution was obtained at $100 \%$ humidity in the scan head compartment. Unfortunately, after a few weeks of operation at this high humidity, the scan head failed. Imaging of polypropylene was also carried out in the NanoScope liquid cell using water. The image quality obtained in the liquid cell was poorer than those obtained in air.

Samples were characterized by performing wide-angle X-ray scattering (WAXS) and differential scanning calorimetry (d.s.c.). WAXS experiments were carried out on isotropic and oriented samples using a Siemens D-500 instrument and a Huber texture goniometer. Pole figures of anisotropic specimens, which were calculated from $\mathrm{X}$-ray intensities, showed a uniaxial texture with chains oriented in the stretching direction. According to results of X-ray diffraction measurements, the polymer contained predominantly the type I monoclinic (or $\alpha$ ) crystal modification in both the unoriented and oriented samples ${ }^{17,18}$. D.s.c. measurements were carried out using a Perkin-Elmer DSC 7 device at $10^{\circ} \mathrm{C} \mathrm{min}^{-1}$ heating rate. The endothermic peak at $161.5^{\circ} \mathrm{C}$ was identified as the melt peak temperature, which agrees well with litcrature data ${ }^{19}$ for $\alpha$-PP. D.s.c. traces of isotropic samples showed a shoulder at $\sim 153^{\circ} \mathrm{C}$. For oriented samples this shoulder became a well pronounced peak. No shift was detected in the position of the melt peak endotherm for the oriented samples.

\section{RESULTS AND DISCUSSION}

AFM images of oriented i-PP on the micrometre scale showed microfibrils with a typical average diameter of $100-150 \mathrm{~nm}$, and with an axis aligned predominantly in the direction of orientation. Figure $l$ is a typical image

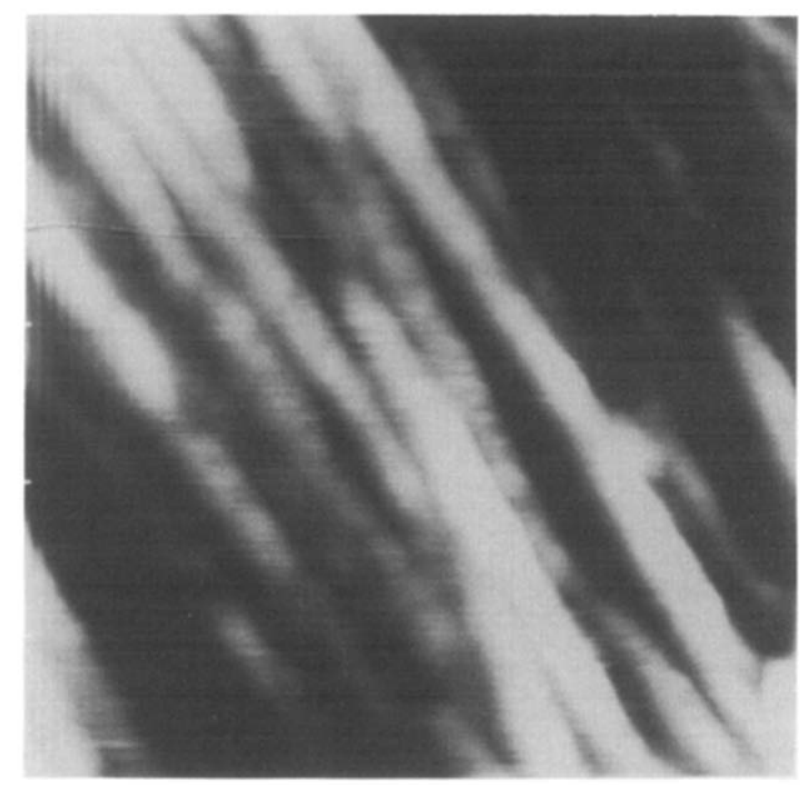

Figure 1 AFM image of microfibrils of oriented polypropylene. Image size: $1000 \mathrm{~nm} \times 1000 \mathrm{~nm}$ 

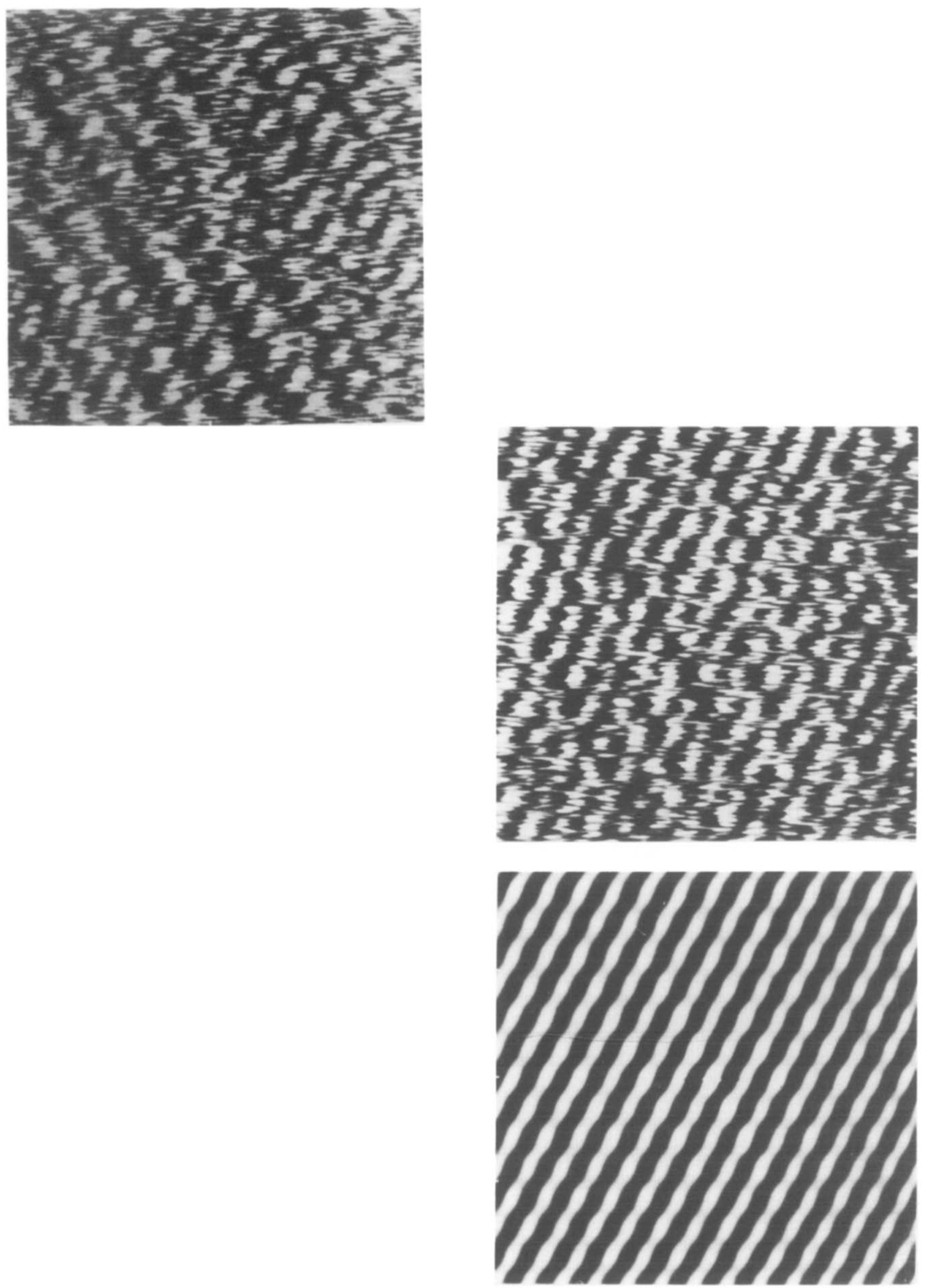


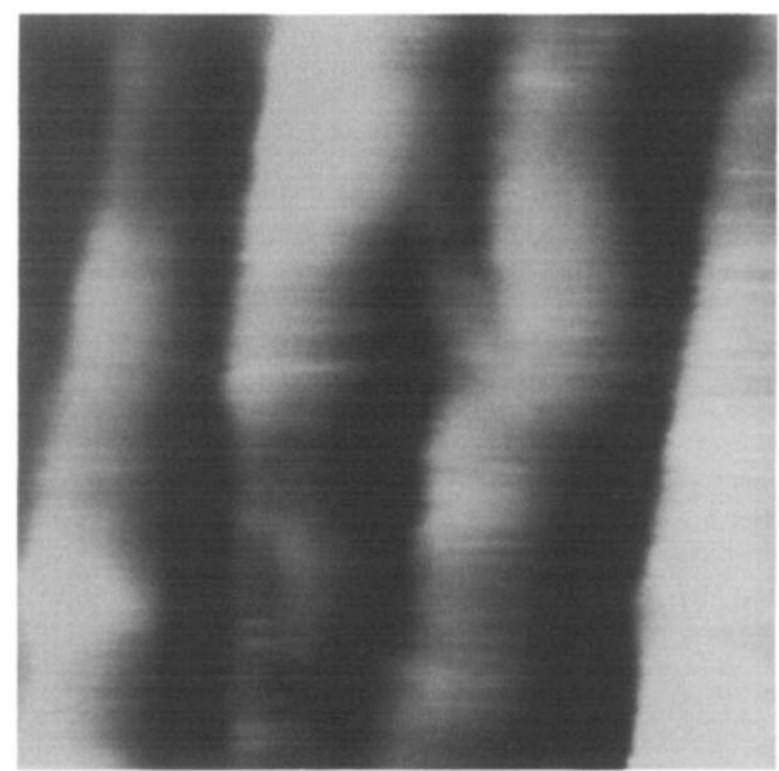

Figure 4 AFM image of the microfibrils $(500 \mathrm{~nm} \times 500 \mathrm{~nm})$ with a perpendicular fibre-axis alignment to the scan direction

elements on the neighbouring chains, which correspond to this structure, can be connected by two straight lines: one making an angle of $40^{\circ}$ with the chain direction, and another one at a $60^{\circ}$ inclination with respect to the chain at the opposite side of the macromolecular backbone. These two directions are marked by overlaid lines in the image shown in Figure $5 b$, which displays the 2D AP of the nanograph shown in Figure $5 a$.

In order to identify these features clearly, the imaged (110) crystal facet of the crystal structure of $\alpha$-PP was visualized on a computer workstation using the Schakal 88 software package ${ }^{21}$. (The analysis of the various packing modes in i-PP has been the subject of numerous papers, the interested reader is referred to ref. 22.) The Schakal software package allows one, among other things, to plot crystal facets of known structures by using space-filling van der Waals radii. For simplicity, we replaced the methyl substituents on the PP chain by chlorine atoms for the computer visualization. The parameters of the crystal structure were taken from the literature ${ }^{17}$. The packing of atoms in the $(110)$ crystal facet is shown in Figures $6 a$ and $b$. The large spheres at the front of the computer-visualized structure represent the $\mathrm{Cl}$ atoms (i.e. they correspond to the $\mathrm{CH}_{3}$ groups), while the smaller, filled and the small empty spheres refer to $\mathrm{C}$ and $\mathrm{H}$ atoms, respectively. Figures $6 a$ and $b$ both show four chains. If one cleaves the crystal in the (1 10$)$ plane, then the symmetry and the packing of the methyl groups in the two cleaved free surfaces correspond to the patterns shown in Figures $6 a$ and $b$. The pattern of the methyl groups in Figures $6 a$ and $b$ form mirror images with respect to a plane parallel to the plane of the drawing. It is well known that in the $\alpha$ form of i-PP, left- and right-handed helices of the PP chains are packed in an alternating manner, and thus the chains form enantiomorphic pairs that face each other. In Figure $6 a$, proceeding from left to right, the first chain corresponds to a right-handed, the second to a left-handed helix, and so on. In this crystal facet, seen from the perspective shown in Figure $6 a$, the $\mathrm{CH}_{3}$ groups form a double column and rhombus-like patterns for the right-handed helices of the same chain, and a single vertical column along the chain for the left-handed helices. In Figure $6 b$ the handedness of the helices from left to right is opposite to the situation depicted in Figure $6 a$. In this case the lozenge-like patterns of the $\mathrm{CH}_{3}$-substituting $\mathrm{Cl}$ atoms refer to left-handed, and the single-column vertical patterns of the $\mathrm{CH}_{3}$ groups to right-handed helices, respectively.

In Figure 7 a line plot of the computer-visualized structure (Figure $6 a$ ) is depicted. Two rows of methyl groups, formed at $40^{\circ}$ and $60^{\circ}$ on the opposite sides of the main chain, are marked by the straight lines. These inclination angles with respect to the main chain correspond exactly to the angles observed on the AFM images. The row of methyl groups at $60^{\circ}$ to the right of the main chain corresponds to the $\left[\begin{array}{lll}1 & 1 & 2\end{array}\right]$ crystallographic
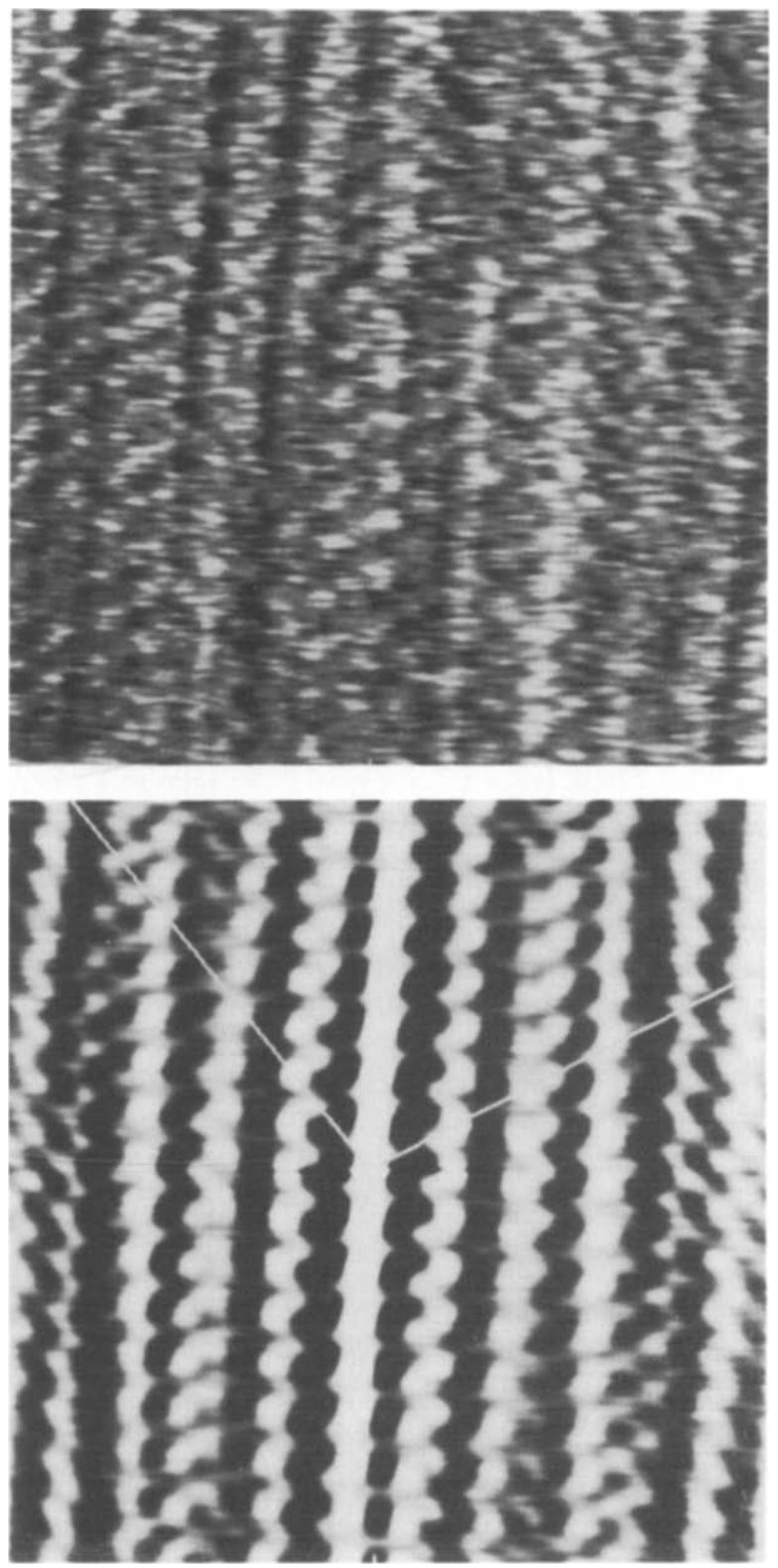

Figure 5 (a) A $10 \mathrm{~nm} \times 10 \mathrm{~nm}$ nanograph obtained on the surface of a microfibril shown in Figure 4. The polypropylene chains, as well as the methyl groups attached to the polymer backbone, are clearly resolved. Twisting of polymer chains, and a paracrystalline disorder can also be seen. (b) Two-dimensional autocorrelation pattern of the AFM image shown in (a) 

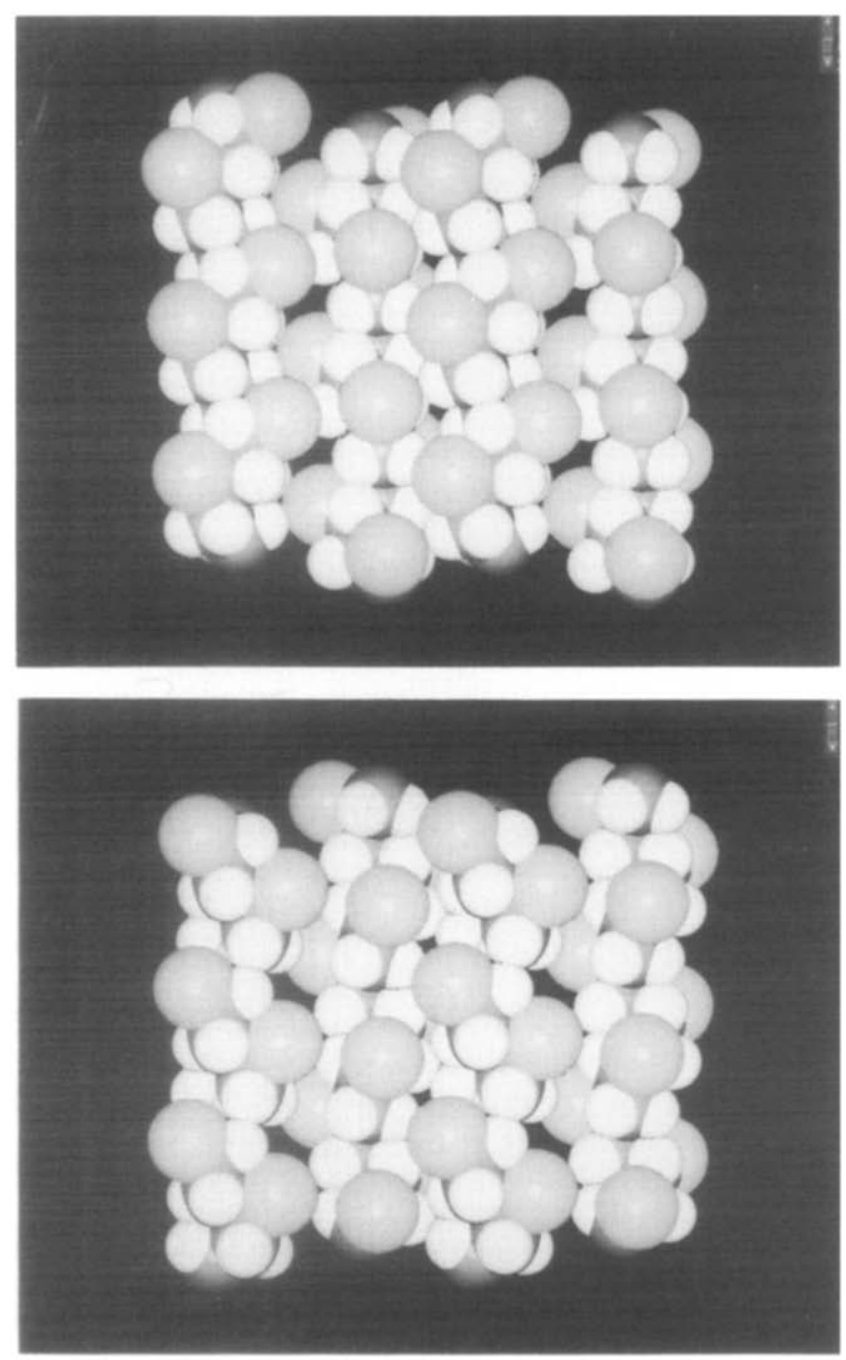

Figure 6 (a) Computer visualization of the molecular packing in the (1 1 0) crystal facet. (b) Computer visualization of the molecular packing in the (110) crystal facet, mirror image of the packing of methyl groups shown in (a) with respect to a plane parallel to the plane of the figure

[1 $\overline{1} 4]$

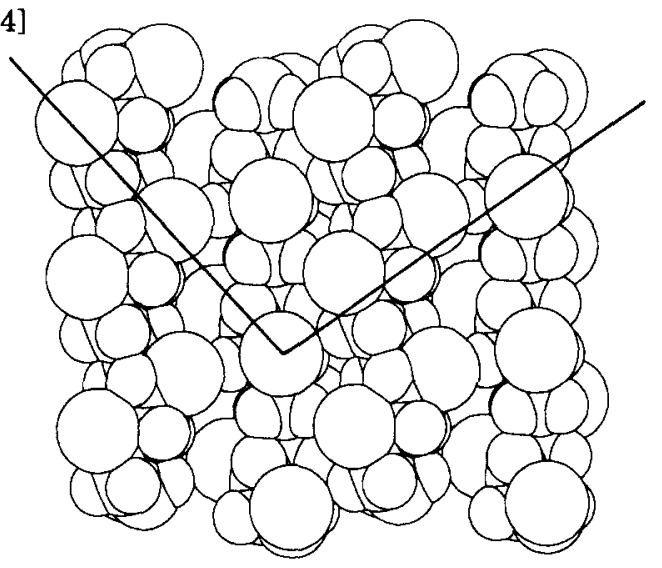

Figure 7 Chain packing corresponding to the structure shown in Figure $6 a$. Lines at $40^{\circ}$ left and $60^{\circ}$ right of the chain direction, respectively, show the rows of methyl groups which were captured on the AFM images

direction, while the row at $40^{\circ}$ at the left of the backbone to the [ 1114$]$ crystallographic direction, respectively. (With the parameters of the crystal structure taken from ref. 17 the exact numerical value of these angles is $59.4^{\circ}$ and $40.19^{\circ}$, respectively.) It is to be expected that the $\mathrm{CH}_{3}$ groups in the scanned facet of the i-PP crystals 'stick out' of the scanned surface and thus give the primary contribution to the contact forces measured in the AFM experiment. Thus, when imaging crystalline PP, different patterns formed by $\mathrm{CH}_{3}$ groups are visualized. Comparing Figure 5b, Figure 7 and the nanostructure obtained by AFM, the assignment of the features, which make an angle of $60^{\circ}$ with the polymer main chain, is obvious: the 'ridges' on the AFM image clearly correspond to linear patterns formed from neighbouring methyl groups. In Figures $3 a$ and $b$, for example, rows of $\mathrm{CH}_{3}$ groups at $60^{\circ}$ at the right of the main chain are observed. This means that the helices depicted in this image with two columns of methyl groups must be right-handed, as shown in Figure 6a. In the mirror image of this facet, the corresponding row of the $\mathrm{CH}_{3}$ groups would be located at $60^{\circ}$ to the left of the polymer backbone. In this case the other row formed of neighbouring methyl groups would be at $40^{\circ}$ to the right of the main chain, just like the images discussed in our previous communication ${ }^{11}$. Nanographs with this characteristic correspond to visualization of left-handed helices with double columns of methyl groups forming the lozenge patterns.

Packing distances were quantitatively determined from a large number of AFM images and the results were then compared with the expected data. A chain-chain packing of $1.08( \pm 0.04) \mathrm{nm}$, and a periodicity in the chain direction of $0.65( \pm 0.06) \mathrm{nm}$ were observed for images with a fibre (or chain) axis at $60^{\circ}$ to the scan direction. The uncertainties of the values quoted in the brackets refer to the standard deviation of the 19 scans which were evaluated. For the images with $95^{\circ}$ clockwise inclination to the scan direction the corresponding values were 1.08 $( \pm 0.03) \mathrm{nm}$ and $0.63( \pm 0.07) \mathrm{nm}$, respectively. These results were obtained from the evaluation of 10 different images. Based on the crystal structure ${ }^{17}$ determined by $\mathrm{X}$-ray diffraction, the expected values are $0.65 \mathrm{~nm}$ for the repeat unit in the chain direction, and $1.10 \mathrm{~nm}$ for the packing distance in the (110) facet. Thus, for the $\alpha$-form of $\mathrm{i}-\mathrm{PP}$, there is excellent agreement between the expected parameters of the crystal structure and the AFM data given in this paper.

It is important to mention that all the AFM images which yielded molecular resolution in this study showed the $(110)$ facet. The crystal structure was probably cleaved in this plane because the corresponding set of crystal planes have a relatively large packing distance, and the packing of molecules within the (110) facet is denser than within other planes.

It is interesting to compare the 2D Fourier-filtered image with the 2D AP. In Figure 8 we depicted the two-dimensional Fourier spectrum of the nanograph shown in Figure 5a. The reconstructed Fourier-filtered image is displayed in Figure 9. This was obtained by the reconstruction of the image, and by keeping the Fourier components framed in the boxes in Figure 8.

The raw data image in Figure $5 a$, the $2 \mathrm{D}$ AP in Figure $5 b$, and the Fourier-filtered image in Figure 9 clearly show identical features, although, as expected, the values of the $F(x, y)$ contact force function have distinct differences. For example, in the 2D AP, the nearly vertical column of methyl groups at the centre of the image has a significantly higher 'intensity' than the surroundings. This is clearly introduced by the calculations of the 2D 


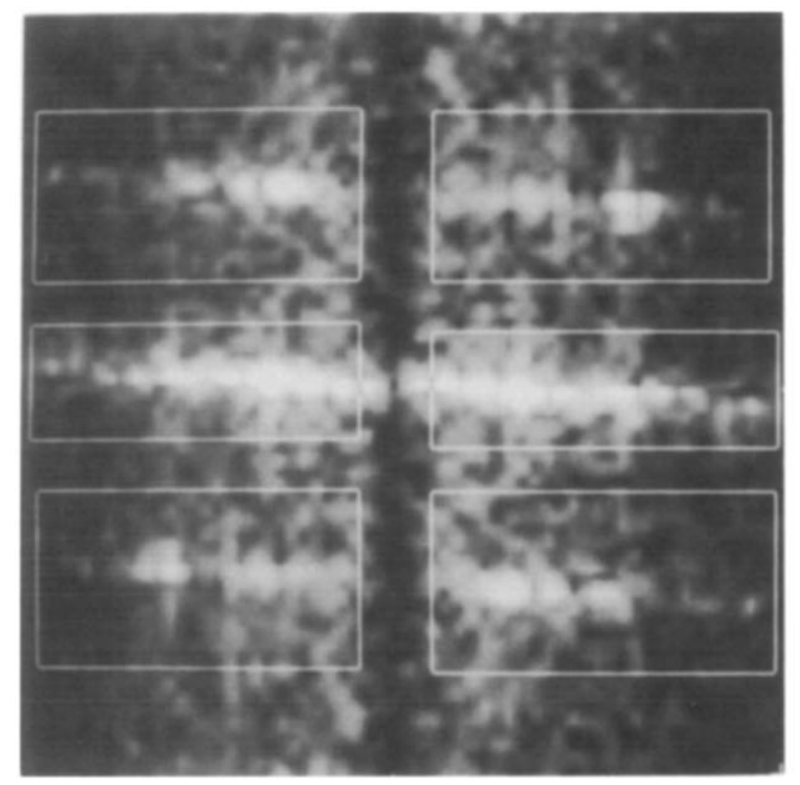

Figure 8 Two-dimensional Fourier-spectrum of the image shown in Figure $5 a$

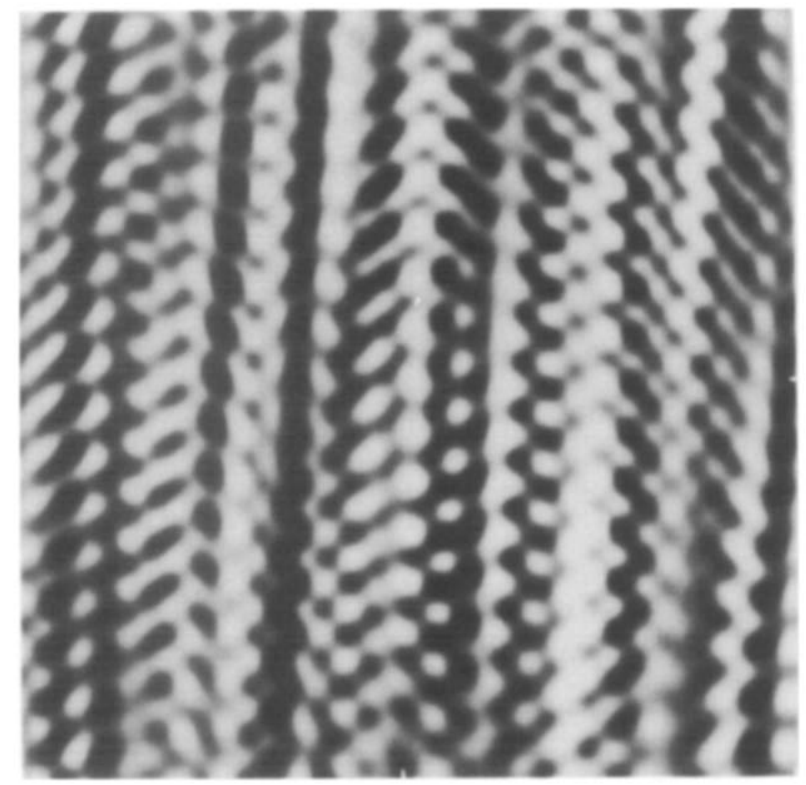

Figure 9 Fourier-filtered image of the scan captured in Figure 5a, calculated by keeping the spectral intensities within the selected areas shown in Figure 8

AP. As all three images show, the chains are packed in a distorted lattice which includes twisted macromolecules. The disorder observed resembles a paracrystalline lattice, and thus corresponds to a lattice distortion of the second kind $^{23}$. If this disorder was eliminated by a careful orientation process, the mechanical performance of the oriented material could be significantly improved.

Finally, in Figure 10 we show a smaller area of $2.7 \mathrm{~nm} \times 2.7 \mathrm{~nm}$ cut from Figure 9. Again, the bright spots on the AFM image correspond to the $\mathrm{CH}_{3}$ groups. It is possible to identify the handedness of the helices which expose the double columns of the methyl groups (those that form the lozenge patterns) by comparing the packing of the methyl groups in the image with the crystal structure shown in Figure $6 a$. For example, the first two vertical columns from the left in both Figure $6 a$ (model) and Figure 10 (AFM image) correspond to methyl groups attached to a right-handed helix. The third single vertical column at $1 \mathrm{~nm}$ from the left edge in Figure 10 therefore must correspond to a left-handed helix, the next two columns to a right-handed helix, and so on. The distances between the vertical columns of the methyl groups can be used to decide which column of the $\mathrm{CH}_{3}$ groups belongs to left- and which two columns to right-handed helices. In Figure 10 the distance between the third and the fourth columns is smaller than the separation between the first and the second columns. The distance in the (110) facet is the shortest between a single column of a left-handed helix and the neighbouring column of the double-column pattern of the methyl groups, attached to the neighbouring right-handed helix (see Figure 6a). Therefore, the image captured in Figure 10 can be attributed to a pattern shown in Figure $6 a$.

At a first glance, it might seem that in Figure 10 the two vertical rows at $1 \mathrm{~nm}$ and $1.2 \mathrm{~nm}$ from the left edge 'stick out' more than the neighbouring ones. This speculation, however, has no experimental support, because the force coordinate $F(x, y)$ in filtered AFM images usually includes artificial contributions (introduced by the filtering manipulation) which increase or decrease the intensity values. We believe that at this stage it is still beyond our reach to analyse quantitatively the surface pattern of atoms or groups of atoms in the surface-perpendicular direction, i.e. a 'depth of field' analysis of AFM images is still speculative.

In conclusion, the AFM was successfully used in this study to image individual methyl groups attached to the main chain of a commercial, isotactic polypropylene. Cleaved samples used in this study were obtained by microtoming of oriented polymeric rods. Cleavage occurred in the (110) facet, presumably because of the large interplain separation distance and the high packing density in this crystal plane. Left- and right-handed helices were distinguished, and structural disorder was oberved on the AFM nanographs, which have unprecedented image quality. These results were achieved by carefully controlling air humidity in the microscope

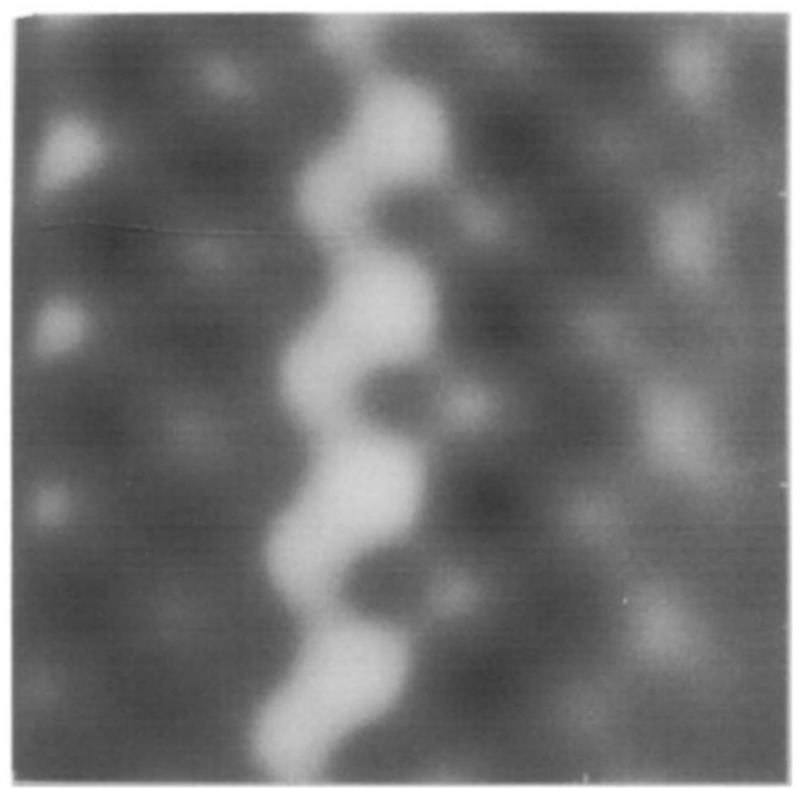

Figure 10 AFM image of a $2.7 \mathrm{~nm} \times 2.7 \mathrm{~nm}$ area displaying methyl groups, and right- and left-handed helices of polypropylene 
compartment. The results also show that studies of structural disorder of oriented polymers in the crystalline phase are possible at the molecular level. It is likely that the AFM will be utilized in the future to understand the deformation mechanism of oriented polymers at the molecular level, and to optimize the end-use performance of anisotropic plastics.

\section{ACKNOWLEDGEMENTS}

Financial support by the Ontario Centre for Materials Research is gratefully acknowledged. The authors thank the Polymer Institute of the Swiss Federal Institute of Technology (ETH-Zürich) for the use of the tensile test instrument and the X-ray diffractometer, and Professor G. C. Rutledge (MIT) for his help with the computer visualization work.

\section{REFERENCES}

1 Binnig, G., Quate, C. F. and Gerber, C. Phys. Rev. Lett. 1986, 56,930

2 Binnig, G. and Rohrer, H. Rev. Mod. Phys. 1987, 59, 615

3 Rugar, D. and Hansma, P. Physics Today 1990, 43, 23

4 For a general review see e.g. Sarid, D. 'Scanning Force Microscopy With Applications to Electric, Magnetic, and Atomic Forces', Oxford University Press, Oxford, 1991
5 Marti, O., Ribi, H. O., Drake, B., Albrecht, T. R., Quate, C. F. and Hansma, P. K. Science 1988, 239, 50

6 Magonov, S. N., Quarnström, K., Elings, V. and Cantow, H.J. Polym. Bull. 1991, 25, 689

7 Lotz, B., Wittmann, J. C., Stocker, W., Magonov, S. N. and Cantow, H.-J. Polym. Bull. 1991, 26, 209

8 Meyer, E., Howald, L., Overney, R. M., Heinzelmann, H., Frommer, J., Güntherodt, H.-J., Wagner, T., Schier, H. and Roth, S. Nature 1991, 349, 398

9 Snétivy, D., Yang, H. and Vancso, G. J.J. Mat.Chem. 1992, 2, 891

10 Snétivy, D. and Vancso, G. J. Macromolecules 1992, 25, 3320

11 Snétivy, D., Guillet, J. E. and Vancso, G. J. Polymer 1993, 34, 429

12 Snétivy, D., Vancso, G. J. and Rutledge, G. C. Macromolecules 1992, 25, 7037

13 Magonov, S. N., Kempf, S., Kimmig, M. and Cantow, H.-J. Polym. Bull. 1991, 26, 715

14 Patil, R., Kim, S. J., Smith, E., Reneker, D. H. and Weisenhorn, A. L. Polym. Commun. 1990, 31, 455

15 Snétivy, D. and Vancso, G. J. Polymer 1992, 33, 432

16 Rutledge, G. C. and Suter, U. W. Macromolecules 1991, 24, 1921

17 Natta, G., Corradini, P. and Cesari, M. Rend. Atti Accad. Nazl. Lincei 1956, 21, 365

18 Turner-Jones, A., Aizlewood, J. M. and Beckett, D. R. Makromol. Chem. 1964, 75, 134

19 Wyckoff, H. W. J. Polym. Sci. 1962, 62, 83

20 NanoScope II User's Manual, Digital Instruments, Santa Barbara, 1989

21 Institute of Crystallography, University of Freiburg, Germany, 1988

22 Lotz, B. and Wittmann, J. C. J. Polym. Sci., Polym. Phys. Edn $1986,24,1541$

23 Kakudo, M. and Kasai, N. 'X-Ray Diffraction by Polymers', Kodansha-Elsevier, Amsterdam, 1972 